\title{
Proteomic and metabolomic analyses provide insight into the off-flavour of fruits from citrus trees infected with 'Candidatus Liberibacter asiaticus'
}

\author{
Lixiao Yao ${ }^{1,2}$, Qibin Yu ${ }^{2}$, Ming Huang ${ }^{2}$, Weilun Hung ${ }^{2}$, Jude Grosser ${ }^{2}$, Shanchun Chen ${ }^{1}$, Yu Wang ${ }^{2}$ and \\ Frederick G. GmitterJr. $\mathbb{D}^{2}$
}

\begin{abstract}
Orange fruit from trees infected by 'Candidatus Liberibacter asiaticus' (CaLas) often do not look fully mature and exhibit off-flavours described as bitter, harsh, and metallic rather than juicy and fruity. Although previous studies have been carried out to understand the effect of CaLas on the flavour of orange juice using metabolomic methods, the mechanisms leading to the off-flavour that occurs in Huanglongbing (HLB)-symptomatic fruit are not well understood. In this study, fruits were collected from symptomatic and healthy Valencia sweet orange (Citrus sinensis) trees grafted on Swingle (C. paradisi X Poncirus trifoliata) rootstock. Isobaric tags for relative and absolute quantification (iTRAQ) and gas chromatography-mass spectrometry (GC-MS) were used to measure the proteins, sugars, organic acids, amino acids, and volatile terpenoids. The results showed that most of the differentially expressed proteins involved in glycolysis, the tricarboxylic acid (TCA) cycle and amino-acid biosynthesis were degraded, and terpenoid metabolism was significantly downregulated in the symptomatic fruit. Valencene, limonene, 3-carene, linalool, myrcene, and aterpineol levels were significantly lower in fruit from CaLas-infected trees than from healthy trees. Similar phenomena were observed for sucrose and glucose. Our study indicated that off-flavour of symptomatic fruit was associated with a reduction in the levels of terpenoid products and the downregulation of proteins in glycolysis, the TCA cycle, and the terpenoid biosynthesis pathway.
\end{abstract}

\section{Introduction}

In Florida, the production of sweet oranges dropped from 240 million boxes in 2004 to 45 million in 2018, substantially because of the most devastating citrus disease named Huanglongbing (HLB) (Florida Citrus Commission: http://www.floridacitrus.org). 'Candidatus Liberibacter asiaticus' ( $\mathrm{Ca}$ Las) is the main pathogen that induces HLB and is naturally transmitted via the Asian citrus psyllid, Diaphorina citri, or by grafting with infected scions. CaLas can be present in leaves, bark, roots, flowers,

\footnotetext{
Correspondence: Frederick G. Gmitter Jr. (fgmitter@ufl.edu)

${ }^{1}$ Citrus Research Institute, Southwest University, Chongqing, China

${ }^{2}$ Citrus Research and Education Center, University of Florida, Lake Alfred, FL

33850, USA
}

and fruit, and the leaf midribs are usually used for detecting the pathogen ${ }^{1,2}$.

HLB not only reduces the quantity of citrus fruit but also affects the quality of fruit and orange juice. HLBimpacted fruits are small, asymmetrical, and lopsided, and have small or aborted seeds. The axis of symptomatic fruit becomes bent, and the central vascular bundles in fruit become deep yellow-orange to brown ${ }^{3}$.The fruit surfaces can be substantially green even at fruit maturity, and may 'break' only on the stem end. Hence, HLB is also called 'citrus greening'. The juice of HLB-impacted mature fruit is lower in percentage and soluble solid content, higher in acidity, has a bitter, harsh, metallic taste, and numerous off-flavours ${ }^{4}$. 
In an attempt to reveal how the flavour of HLB orange juice is changed, the chemical compound composition of diseased fruit were examined by several research groups ${ }^{5-9}$. Lower amounts of sugars and higher amounts of titratable acid are found in the HLB-affected fruits, resulting in a lower soluble solid/acid ratio ${ }^{5-7}$. A lower soluble solid/acid ratio often indicates that the symptomatic fruits are bitter and sour and similar to an unripe product. Bitter principles, such as limonin, are elevated greatly in juice from the symptomatic fruit. However, none of the limonin levels observed exceed those of the average bitterness threshold in HLB juice ${ }^{5,8,9}$. The investigation of orange juice from CaLas-infected and CaLas-free trees by gas chromatography-mass spectrometry (GC-MS) showed that symptomatic Valencia orange juice has similar total volatiles to those of the healthy control juice but only $40 \%$ of the total esters, $48 \%$ of the total aldehydes, and $33 \%$ of the total sesquiterpenes of the control juice ${ }^{5}$. The concentration of valencene is also $50-67 \%$ lower in juice from symptomatic fruit compared with that of the healthy control. The levels of esters, especially ethyl butanoate and ethyl 2-methylbutyrate, and soapy-waxy alkanals, such as octanal and decanal, are significantly lower in HLB-impacted orange juice $^{5,8}$, whereas the levels of juice terpenes (such as $\gamma$ terpinene and $\alpha$-terpinolene), alcohol, green aldehydes (such as hexanal), and degradation compounds of limonene and linalool (such as $\alpha$-terpineol) are higher in symptomatic juice than those in the control ${ }^{8}$. Therefore, the previously reported off-flavour associated with HLB-symptomatic juices is likely not bitterness alone but is apparently the result of the juices being less sweet and more sour due to lower sugar and higher acid levels and an imbalance of certain volatile compounds ${ }^{6,8,9}$.

Research has been conducted to identify key genes, proteins, and organic products induced by $C a$ Las in leaf tissues through transcriptomic, proteomic, or metabolomic studies ${ }^{10-14}$. These studies have shown that the vital biological pathways and processes, such as cell defence, transport, photosynthesis, carbohydrate metabolism, and hormone metabolism, are affected by the disease. HLBimpacted fruit has a modified carbohydrate and phytohormone balance ${ }^{15}$. Two research teams analysed the whole-genome expression of fruit peel from CaLasinfected trees. They found that the transcription of genes involved in the light reactions of photosynthesis and in ATP synthesis was enhanced, and the proteins involved in degradation and misfolding processes were activated under the HLB stress. At the same time, HLB also strongly affected source-to-sink communication pathways, including sucrose and starch metabolism and hormone synthesis and signalling ${ }^{1,16}$.

Proteomics and metabolomics, each with its own advantages, have different analytical objects. Proteomics aims to detail an overall and comprehensive understanding of protein characteristics directly relevant to physiological phenotypes and underlying processes. Metabolomics aims to analyse quantitatively all low molecular weight biomolecules that are the end products of reactions mediated by proteins during a specific physiological period. The integration of proteomic and metabolomic analyses in one study could create a more comprehensive overview of changes in protein expression and metabolite composition and aid the understanding of the metabolic and physiological changes of HLB-affected fruits. In this study, we used isobaric tags for relative and absolute quantification (iTRAQ) and GC-MS to measure the proteins and metabolic compounds of the juice sacs from symptomatic and healthy fruits. The relationship between metabolic compounds and differentially expressed proteins in pulp of HLB-affected and healthy fruit was assessed, revealing the molecular mechanisms determining off-flavour in symptomatic fruits.

\section{Results \\ CaLas detection and HLB-affected fruit symptoms}

Healthy and CaLas-infected trees were selected from a commercial citrus grove and confirmed by quantitative PCR (qPCR) with CaLas-specific primers ${ }^{17}$. The leaves from healthy trees were PCR negative $(\mathrm{Ct}>32)$ and those from symptomatic trees were PCR positive $(\mathrm{Ct} \leq 32)$. However, $\mathrm{CaLas}$ could not be detected in fruit pulp from either healthy or infected trees. Obvious phenotypic differences were found between HLB-affected and healthy fruits; symptomatic fruits were smaller and greener than the healthy fruits (Fig. 1). The soluble solids content of fruit from CaLas-infected trees $\left(9.52{\left.\mathrm{~g} 100 \mathrm{~g}^{-1}\right) \text { was }}\right.$ decreased compared with that of healthy fruit (11.24 $\mathrm{g}$ $100 \mathrm{~g}^{-1}$ ), whereas the acid content of fruit from CaLasinfected trees $(0.82 \%)$ was increased compared with that of healthy fruit $(0.70 \%)$.

\section{Overview of the downregulated proteins in primary and secondary metabolism}

In total, 123 differentially expressed proteins were involved in the biological processes of sugar breakdown and glycolysis, the Calvin cycle, the tricarboxylic acid cycle (TCA), amino-acid synthesis and breakdown, UDPsugar, terpenes, and the phenylpropanoid metabolic pathway (Fig. 2). There were 45 downregulated proteins involved in sugar and organic acid metabolism (Table 1, Fig. 3), 22 downregulated proteins involved in amino-acid metabolism (Table 2), five differentially expressed proteins involved in terpenoid metabolism (Table 3), and three downregulated proteins involved in pectin metabolism (Fig. 4). The expression of 45 enzymes involved in carbohydrate metabolism was downregulated in fruit from trees infected with $\mathrm{CaLas}$, including 12 oxidoreductases (EC 1 family), 12 transferases (EC 2 family), 

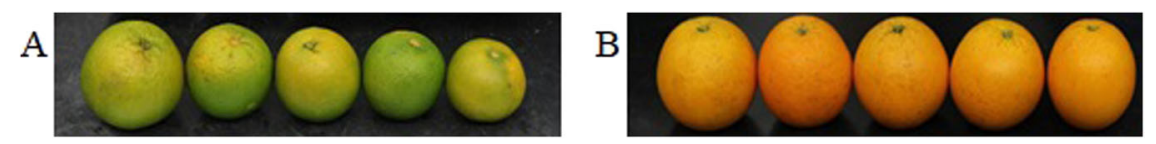

Fig. $1 \mathrm{HLB}$-symptomatic (A) and healthy (B) fruits from Valencia grafted on Swingle rootstock

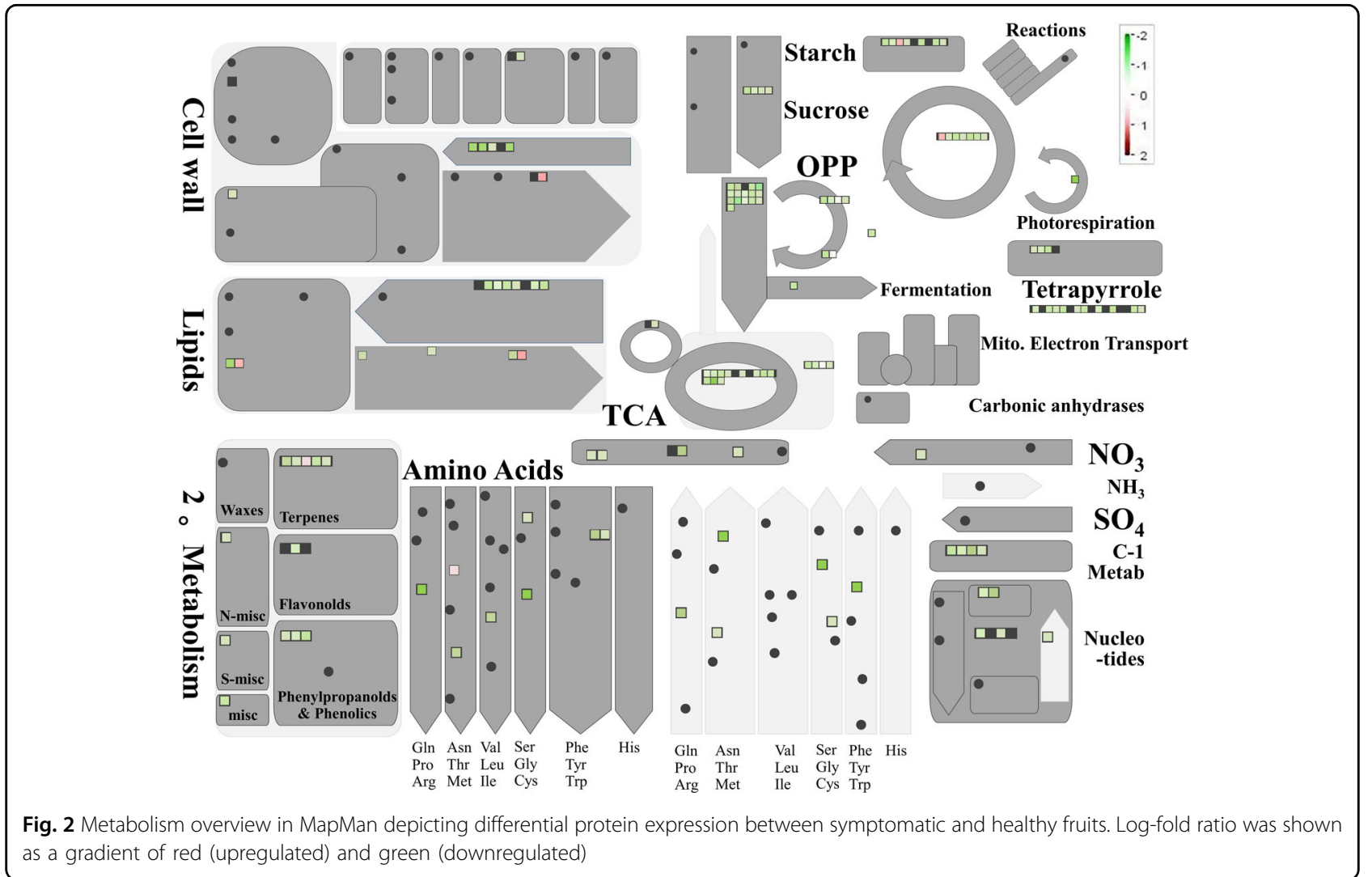

2 hydrolases (EC 3 family), 12 lyases (EC 4 family), and 7 isomerases (EC 5 family) (Table 1). The expression of 22 enzymes involved in amino-acid metabolism was downregulated in fruit from trees infected with CaLas, including eight oxidoreductases (EC 1 family), five transferases (EC 2 family), three hydrolases (EC 3 family), five lyases (EC 4 family), and one ligase (EC 6 family) (Table 2). In the terpenoid biosynthesis process (Table 3 , Fig. 5), there were two downregulated oxidoreductases (EC 1 family), two downregulated transferases (EC 2 family), and one upregulated isomerase (EC 5 family) in fruit from trees infected with $\mathrm{Ca}$ Las. Regarding pectin metabolism, the downregulated proteins were pectin methylesterase (gi|641854939), UDP-D-glucose 4-epimerase (gi|568845013), and UDP-glucose dehydrogenase (gi|641853915) (Table 1, Fig. 4).

\section{Downregulated proteins involved in sugar and organic} acid metabolism and homeostasis

Most of the proteins included in sucrose degradation and glycolytic pathways were downregulated when compared with those in healthy mature fruit. Sucrose is degraded by sucrose synthase (SUS) to generate fructose and UDP-glucose. The expression of SUS (gi|641868466) was decreased in fruit from trees infected with $\mathrm{CaLas}$. Fructokinase (gi|568849997 and gi|641841250) and pyrophosphate:fructose-6-phosphate 1-phosphotransferase (gi|641866581), which catalyse the phosphorylation of fructose, were also reduced on the protein level in symptomatic fruits. UDP-glucose can be converted into glucose-1P catalysed by UDP-glucose pyrophosphorylase (gi|641838381) and UDP-sugar pyrophosphorylase (gi| 568837718), which is involved in the glycolysis process 
Table 1 Downregulated proteins involved in carbohydrate metabolism in symptomatic versus healthy Valencia orange fruit

\begin{tabular}{|c|c|c|c|c|c|c|}
\hline Citrus ID & TAIR code & Protein symbol & Description & Enzyme no. & SS:HS & $P$-value \\
\hline gi|568823164 & AT1G54220 & MTE2-3 & Mitochondrial pyruvate dehydrogenase subunit 2-3 & EC 2.3.1.12 & 0.62 & $1.34 \mathrm{E}-03$ \\
\hline gi|568828917 & AT2G01140 & FBA3 & Fructose-bisphosphate aldolase 3 & EC 4.1.2.13 & 0.57 & 1.16E-04 \\
\hline gi|568831440 & AT5G40760 & G6PD6 & Glucose-6-phosphate dehydrogenase 6 & EC 1.1.1.49 & 0.52 & $1.55 \mathrm{E}-02$ \\
\hline gi|568831566 & AT3G01850 & F28J7.18 & Ribulose-phosphate 3-epimerase & EC 5.1.3.1 & 0.65 & 1.13E-02 \\
\hline gi|568833896 & AT1G71170 & F23N20.16 & 6-Phosphogluconate dehydrogenase-like protein & EC 1.1.1.44 & 0.56 & 1.83E-04 \\
\hline gi|568836722 & AT4G37870 & PCK1 & Phosphoenolpyruvate carboxykinase 1 & EC 4.1.1.49 & 0.58 & 2.19E-02 \\
\hline gi|568837718 & AT5G52560 & USP & UDP-sugar pyrophosphorylase & EC 2.7.7.64 & 0.47 & 4.50E-02 \\
\hline gi|568840482 & AT5G38410 & MXI10.13 & Ribulose bisphosphate carboxylase small chain $3 \mathrm{~B}$ & EC 4.1.1.39 & 0.55 & $9.42 \mathrm{E}-03$ \\
\hline gi|568845013 & AT4G10960 & UGE5 & UDP-D-glucose 4-epimerase 5 & EC 5.1.3.2 & 0.51 & 2.93E-02 \\
\hline gi|568849997 & AT3G59480 & FRK3 & Fructokinase 3 & EC 2.7.1.4 & 0.55 & $1.02 \mathrm{E}-04$ \\
\hline gi|568855816 & AT5G03290 & $\mathrm{IDH}-\mathrm{V}$ & $\mathrm{NAD}^{+}$-dependent isocitric dehydrogenase $\mathrm{V}$ & EC 1.1.1.41 & 0.55 & $1.28 \mathrm{E}-03$ \\
\hline gi|568856679 & AT2G36530 & ENO2 & Enolase 2 & EC 4.2.1.11 & 0.62 & $1.85 \mathrm{E}-10$ \\
\hline gi|568864726 & AT1G74030 & ENO1 & Enolase 1 & EC 4.2.1.11 & 0.59 & $3.20 \mathrm{E}-02$ \\
\hline gi|568868208 & AT2G21170 & TPI & Triosephosphate isomerase & EC 5.3.1.1 & 0.58 & 7.45E-04 \\
\hline gi|568870281 & AT3G55440 & TPI & Triosephosphate isomerase & EC 5.3.1.1 & 0.61 & $1.28 \mathrm{E}-02$ \\
\hline gi|568870518 & AT2G29560 & ENOC & Cytosolic enolase 3 & EC 4.2.1.11 & 0.64 & $6.79 \mathrm{E}-03$ \\
\hline gi|568881904 & AT1G56190 & PGK2 & Phosphoglycerate kinase & EC 2.7.2.3 & 0.55 & $8.96 \mathrm{E}-03$ \\
\hline gi|568881906 & AT1G79550 & PGK3 & Phosphoglycerate kinase & EC 2.7.2.3 & 0.55 & $5.80 \mathrm{E}-06$ \\
\hline gi|568883658 & AT1G65930 & $\mathrm{ClCDH}$ & Cytosolic NADP ${ }^{+}$-dependent isocitrate dehydrogenase & EC 1.1.1.42 & 0.58 & 2.47E-02 \\
\hline gi|572152874 & AT1G53310 & PPC1 & Phosphoenolpyruvate carboxylase 1 & EC 4.1.1.31 & 0.62 & 4.57E-04 \\
\hline gi|641829225 & AT3G15020 & $\mathrm{mMDH} 2$ & Mitochondrial malate dehydrogenase 2 & EC 1.1.1.37 & 0.64 & 1.03E-04 \\
\hline gi|641838381 & AT5G17310 & UGP2 & UDP-glucose pyrophosphorylase 2 & EC 2.7.7.9 & 0.57 & $2.46 \mathrm{E}-11$ \\
\hline gi|641838649 & AT1G04410 & c-NAD-MDH1 & Cytosolic-NAD-dependent malate dehydrogenase 1 & EC 1.1.1.37 & 0.64 & 1.10E-04 \\
\hline gi|641839211 & AT5G13420 & TRA2 & Transaldolase 2 & EC 2.2.1.2 & 0.55 & 8.15E-04 \\
\hline gi|641841250 & AT5G51830 & FRK7 & Fructokinase 7 & EC 2.7.1.4 & 0.60 & $3.11 \mathrm{E}-03$ \\
\hline gi|641841327 & AT1G08110 & GLYI2 & Glyoxalase 12 & EC 4.4.1.5 & 0.66 & $3.00 \mathrm{E}-02$ \\
\hline gi|641842093 & AT3G52990 & AT3G52990 & Pyruvate kinase & EC 2.7.1.40 & 0.60 & $1.30 \mathrm{E}-02$ \\
\hline gi|641847603 & AT1G67280 & GLYI6 & Glyoxalase 16 & EC 4.4.1.5 & 0.66 & $1.70 \mathrm{E}-02$ \\
\hline gi|641847704 & AT2G19860 & HXK2 & Hexokinase & EC 2.7.1.1 & 0.64 & $1.68 \mathrm{E}-02$ \\
\hline gi|641849423 & AT4G35260 & $\mathrm{IDH} 1$ & $\mathrm{NAD}^{+}$-dependent isocitrate dehydrogenase 1 & EC 1.1.1.41 & 0.57 & 4.29E-02 \\
\hline gi|641851263 & AT1G68750 & PPC4 & Phosphoenolpyruvate carboxylase 4 & EC 4.1.1.31 & 0.63 & 3.05E-02 \\
\hline gi|641851457 & AT1G13700 & PGL1 & 6-Phosphogluconolactonase 1 & EC 3.1.1.31 & 0.54 & $3.89 \mathrm{E}-03$ \\
\hline gi|641853224 & AT1G48030 & mtLPD1 & Mitochondrial lipoamide dehydrogenase 1 & EC 1.8.1.4 & 0.65 & $5.78 \mathrm{E}-05$ \\
\hline gi|641853915 & AT5G15490 & UGD3 & UDP-glucose dehydrogenase & EC 1.1.1.22 & 0.58 & $2.33 \mathrm{E}-02$ \\
\hline gi|641854939 & AT3G14310 & PME3 & Pectin methylesterase 3 & EC 3.1.1.11 & 0.62 & 7.18E-05 \\
\hline gi|641859572 & AT1G11840 & GLYI3 & Glyoxalase 13 & EC 4.4.1.5 & 0.43 & $2.84 \mathrm{E}-02$ \\
\hline gi|641862435 & AT4G24620 & PGl1 & Plastid phospho-glucose (Glc) isomerase & EC 5.3.1.9 & 0.55 & 7.70E-04 \\
\hline gi|641863965 & AT1G59900 & E1 ALPHA & Pyruvate dehydrogenase complex E1 alpha subunit & EC 1.2.4.1 & 0.61 & 4.08E-03 \\
\hline gi|641864450 & AT5G50850 & MAB1 & Pyruvate dehydrogenase E1 beta & EC 1.2.4.1 & 0.57 & 8.81E-03 \\
\hline
\end{tabular}


Table 1 continued

\begin{tabular}{llllrcc}
\hline Citrus ID & TAIR code & Protein symbol & Description & Enzyme no. & SS:HS & $P$-value \\
\hline gi|641865848 & AT5G42740 & MJB21.12 & Glucose-6-phosphate isomerase & EC 5.3.1.9 & 0.54 & $4.45 E-04$ \\
gi|641866581 & AT1G76550 & F14G6.15 & Pyrophosphateffructose-6-phosphate 1-phosphotransferase & EC 2.7.1.90 & 0.52 & $2.58 E-07$ \\
gi|641867382 & AT4G00570 & NAD-ME2 & NAD-dependent malic enzyme 2 & EC 1.1.1.39 & 0.55 & $2.68 \mathrm{E}-04$ \\
gi|641867393 & AT2G45790 & PMM & Phosphomannomutase & EC 5.4.2.8 & 0.52 & $2.41 \mathrm{E}-03$ \\
gi|641868430 & AT2G47510 & FUM1 & Fumarate hydratase 1 & EC 4.2.1.2 & 0.53 & $7.83 \mathrm{E}-04$ \\
gi|641868466 & AT4G02280 & SUS3 & Sucrose synthase 3 & EC 2.4.1.13 & 0.61 & $4.51 \mathrm{E}-03$ \\
\hline
\end{tabular}

SS symptomatic fruit samples, HS healthy fruit samples

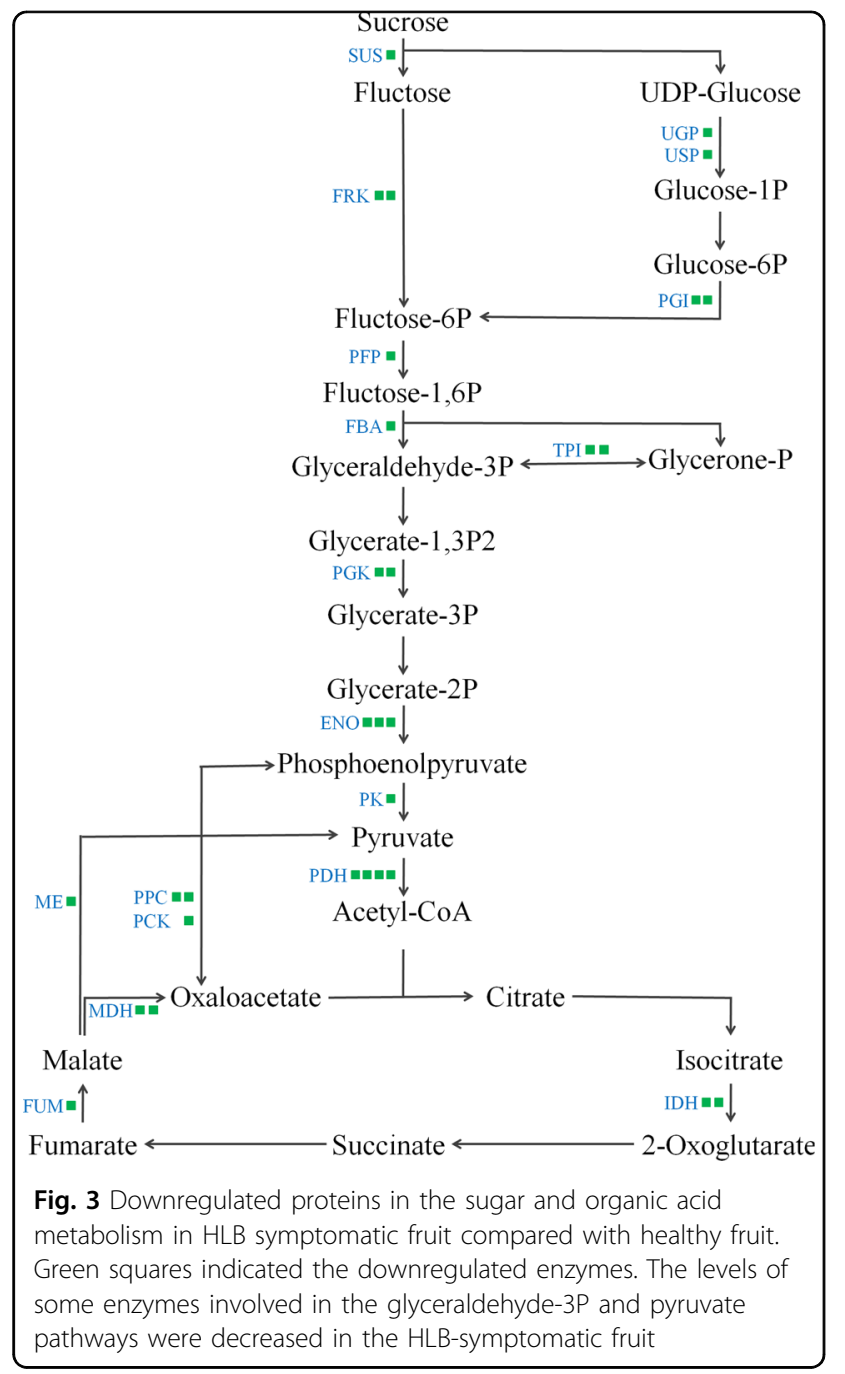

(Fig. 3). It can also be converted into UDP-glucuronate catalysed by UDP-glucose dehydrogenase (gi|641853915), which is involved in the synthesis of lignin (Fig. 4). Three of the enzymes were found at lower levels in diseased fruit than in the healthy control. All homologous proteins of Arabidopsis enolases (gi|568856679, gi|568864726, and gi|568870518) and phosphoglycerate kinases (gi| 568881904 and gi|568881906) related to glycolysis were downregulated in the symptomatic fruit. Two isocitrate dehydrogenases (gi|568855816 and gi|641849423), two malate dehydrogenases (gi|641829225 and gi|641838649), and a fumarate hydratase (gi|641868430) were found at lower levels in the HLB-affected orange fruits than in the healthy control.

Conversely, many proteins associated with pyruvate metabolism were downregulated by $\mathrm{Ca}$ Las. Levels of phosphoenolpyruvate carboxylase (gi|572152874 and gi| 641851263) and carboxykinase (gi|568836722), which are associated with the transformation between phosphoenolpyruvate and oxaloacetate, were decreased. Levels of malic enzyme (gi|641867382), which transforms malate to pyruvate, were reduced in fruit from $\mathrm{Ca}$ Las-infected trees. The pyruvate dehydrogenase enzyme complex connects glycolysis with the TCA cycle. Levels of two of the pyruvate dehydrogenase complex proteins (gi|641863965 and gi|641864450) were reduced, and levels of two other components of the same complex, dihydrolipoamide Sacetyltransferase (gi|568823164) and dihydrolipoamide dehydrogenase (gi|641853224), were downregulated in symptomatic fruit (Table 1).

\section{Changes in sugar, organic acid, and amino-acid metabolism}

The standard products for 8 distinctive sugars, 5 organic acids, and 12 amino acids were run (Table 4). Some tetramethyl silane (TMS) derivatives of the non-volatile products could not be detected using GC-MS, including mannose, rhamnose, sorbitol, oxalic acid, tartaric acid, arginine, cysteine, glutamine, histidine, isoleucine, lysine, methionine, tyrosine, and cystine. Sucrose, fructose, and glucose were the main distinctive sugars in the Valencia pulp, and their concentration was decreased $26 \%, 15 \%$, and $20 \%$, respectively, in symptomatic sweet orange fruit compared with the healthy control. Although some lowcontent monosaccharides such as arabinose (18.88\%), mannitol (38.07\%), and ribose (28.79\%), were increased, the main organic acids, citric acid and malic acid, 
Table 2 Differentially regulated enzymes (proteins) involved in amino-acid metabolism in symptomatic versus healthy Valencia orange fruit

\begin{tabular}{|c|c|c|c|c|c|c|}
\hline Citrus ID & TAIR code & Protein symbol & Description & Enzyme no. & SS:HS & $P$-value \\
\hline gi|568821659 & AT1G80560 & IMD2 & 3-Isopropylmalate dehydrogenase 2 & EC 1.1.1.85 & 0.66 & $3.20 \mathrm{E}-03$ \\
\hline gi|568825237 & AT5G66120 & K2A18.20 & Putative 3-dehydroquinate synthase & EC 4.2.3.4 & 0.55 & 2.42E-04 \\
\hline gi|568825398 & AT2G17630 & PSAT2 & Pyridoxal phosphate-dependent transferase & EC 2.6.1.52 & 0.66 & $2.54 \mathrm{E}-02$ \\
\hline gi|568867337 & AT1G49820 & MTK & 5-Methylthioribose kinase & EC 2.7.1.100 & 0.63 & $7.61 \mathrm{E}-03$ \\
\hline gi|568875049 & AT5G52810 & SARD4 & NAD(P)-binding Rossmann-fold superfamily protein & EC 4.3.1.12 & 0.56 & 4.07E-02 \\
\hline gi|568876134 & AT3G61440 & CYSC1 & Cysteine synthase $\mathrm{C} 1$ & EC 2.5.1.47 & 0.54 & 1.94E-02 \\
\hline gi|568881821 & AT1G79440 & ALDH5F1 & Succinate-semialdehyde dehydrogenase & EC 1.2.1.16 & 0.58 & $1.46 \mathrm{E}-02$ \\
\hline gi|641821618 & AT2G31570 & GPX2 & Glutathione peroxidase 2 & EC 1.11.1.9 & 0.64 & 4.29E-02 \\
\hline gi|641823192 & AT3G17760 & GAD5 & Glutamate decarboxylase 5 & EC 4.1.1.15 & 0.64 & 4.45E-04 \\
\hline gi|641823815 & AT4G13940 & MEE58 & S-adenosyl-L-homocysteine hydrolase & EC 3.3.1.1 & 0.64 & $1.43 \mathrm{E}-03$ \\
\hline gi|641831913 & AT5G48220 & MIF21.11 & Aldolase-type TIM barrel family protein & EC 4.1.1.48 & 0.57 & $1.88 \mathrm{E}-05$ \\
\hline gi|641833609 & AT4G24830 & F617.40 & Argininosuccinate synthase & EC 6.3.4.5 & 0.49 & $3.92 \mathrm{E}-02$ \\
\hline gi|641838358 & AT1G65820 & F1E22.17 & Putative glutathione S-transferase & EC 1.11.1.9 & 0.61 & $2.68 \mathrm{E}-02$ \\
\hline gi|641841873 & AT1G14810 & F10B6.22 & Aspartate semialdehyde dehydrogenase & EC 1.2.1.11 & 0.64 & $3.17 \mathrm{E}-02$ \\
\hline gi|641842227 & AT3G24170 & GR1 & Glutathione-disulphide reductase 1 & EC 1.8.1.7 & 0.50 & 2.37E-02 \\
\hline gi|641848539 & AT4G31870 & GPX7 & Glutathione peroxidase 7 & EC 1.11.1.9 & 0.53 & $2.21 \mathrm{E}-02$ \\
\hline gi|641849692 & AT3G54640 & TSA1 & Alpha-tryptophan synthase & EC 4.2.1.20 & 0.65 & $3.45 \mathrm{E}-02$ \\
\hline gi|641855678 & AT5G43850 & ARD4 & Acireductone dioxygenase 4 & EC 1.13.11.54 & 0.65 & 3.13E-02 \\
\hline gi|641859599 & AT1G11860 & F12F1.30 & Aminomethyltransferase & EC 2.1.2.10 & 0.48 & 3.54E-02 \\
\hline gi|641859748 & AT1G12050 & $\mathrm{FAH}$ & Putative fumarylacetoacetase & EC 3.7.1.2 & 0.54 & 6.07E-04 \\
\hline gi|641862021 & AT1G44820 & T12C22.9 & Peptidase M20/M25/M40 family protein & EC 3.5.1.14 & 0.57 & 2.13E-02 \\
\hline gi|641862593 & AT5G26780 & $\mathrm{SHM} 2$ & Serine hydroxymethyltransferase 2 & EC 2.1.2.1 & 0.57 & $2.13 \mathrm{E}-02$ \\
\hline
\end{tabular}

SS symptomatic fruit samples, HS healthy fruit samples

Table 3 Differentially regulated enzymes (proteins) involved in terpenoid metabolism in symptomatic versus healthy Valencia orange fruit

\begin{tabular}{|c|c|c|c|c|c|c|}
\hline Citrus ID & TAIR code & Protein symbol & Protein description & Enzyme no. & SS:HS & $P$-value \\
\hline gi|568833221 & AT2G02500 & IspD & 2-C-methyl-D-erythritol-4-phosphate cytidylyltransferase & EC 2.7.7.60 & 0.62 & $3.19 \mathrm{E}-03$ \\
\hline gi|568858669 & AT3G63520 & CCD1 & 9-Cis-epoxycarotenoid dioxygenase & EC 1.13.11.51 & 0.57 & 4.05E-02 \\
\hline gi|568869804 & AT5G60600 & IspG & Hydroxy-2-methyl-2-butenyl 4-diphosphate (HMBPP) synthase & EC 1.17.7.1 & 0.63 & 8.95E-03 \\
\hline gi|641835071 & AT5G16440 & $|\mathrm{D}| 1$ & Isopentenyl diphosphate delta-isomerase | & EC 5.3.3.2 & 1.69 & $2.78 \mathrm{E}-05$ \\
\hline gi|641840281 & AT4G17190 & FPS2 & Farnesyl diphosphate synthase & EC 2.5.1.10 & 0.57 & $6.89 \mathrm{E}-05$ \\
\hline
\end{tabular}

SS symptomatic fruit samples, HS healthy fruit samples

decreased $6.50 \%$ and $9.20 \%$, respectively, compared with the healthy control. The low-content organic acids, fumaric acid, quinic acid, and succinic acid, increased $48.06 \%, 20.03 \%$, and $54.82 \%$, respectively, compared with the healthy control. The levels of two acidic amino acids, aspartic acid (3.23\%) and glutamic acid (13.71\%), were decreased in symptomatic fruit. The following neutral amino acids were more highly accumulated in symptomatic fruit: alanine $(10.00 \%)$, asparagine $(128.12 \%)$, glycine $(31.58 \%)$, leucine $(73.66 \%)$, phenylalanine $(79.44 \%)$, proline $(20.57 \%)$, serine $(74.14 \%)$, threonine $(217.79 \%)$, tryptophan (61.83\%), and valine (20.39\%). 


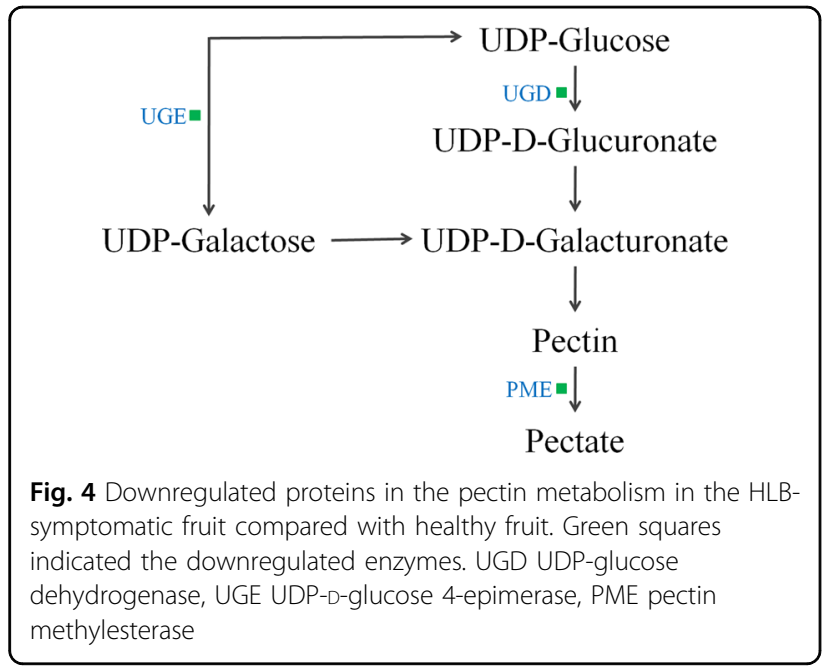

\section{Low levels of terpenoids and proteins involved in terpenoid metabolism}

According to MS peak identification and standard confirmation, six monoterpenoids and one sesquiterpenoid (valencene) were identified in the Valencia pulp. All levels were lower in the symptomatic fruits than those in the healthy fruits: $\alpha$-pinene (77.33\%), 3-carene (87.20\%), myrcene (85.53\%), limonene (82.29\%), linalool (77.57\%), $\alpha$-terpineol (72.16\%), and (+)-valencene (78.66\%) (Table 5).

All isoprenoids are synthesised from isopentenyl diphosphate (IPP) and dimethylallyl diphosphate (DMAPP), the two building blocks, which are produced by the mevalonic acid (MVA) and 2-C-methyl-D-erythritol-4-phosphate (MEP) pathways. 2-C-methyl-D-erythritol-4-phosphate cytidylyltransferase (IspD) catalyses MEP to form 4-diphosphocytidyl-2-C-methyl-D-erythritol (CDP-ME) and is the third enzyme of the MEP pathway. Hydroxy-2-methyl-2-(E)-butenyl 4-diphosphate synthase (IspG) catalyses 2-C-methyl-D-erythritol 2,4-cyclodiphosphate (MEcPP) to form 1-hydroxy-2-methyl-2-butenyl 4diphosphate (HMBPP). That is the penultimate catalysing step of the biosynthesis of IPP and DMAPP through the MEP pathway. The enzyme ispD and ispG (gi|568833221 and gi|568869804) were all downregulated in symptomatic fruit. The levels of farnesyl diphosphate synthase (gi|641840281), which catalyses the rate-limiting step in geranyl pyrophosphate (GPP) and farnesyl pyrophosphate (FPP) biosynthesis, was also reduced in symptomatic fruit (Fig. 5).

\section{Discussion}

A total of 123 differentially expressed proteins were found in primary and secondary metabolism pathways, when comparing HLB-affected fruit with healthy fruit; the fold change threshold was designated as upregulation/ downregulation $=1.5 / 0.67$. The 1.5 -fold change has been

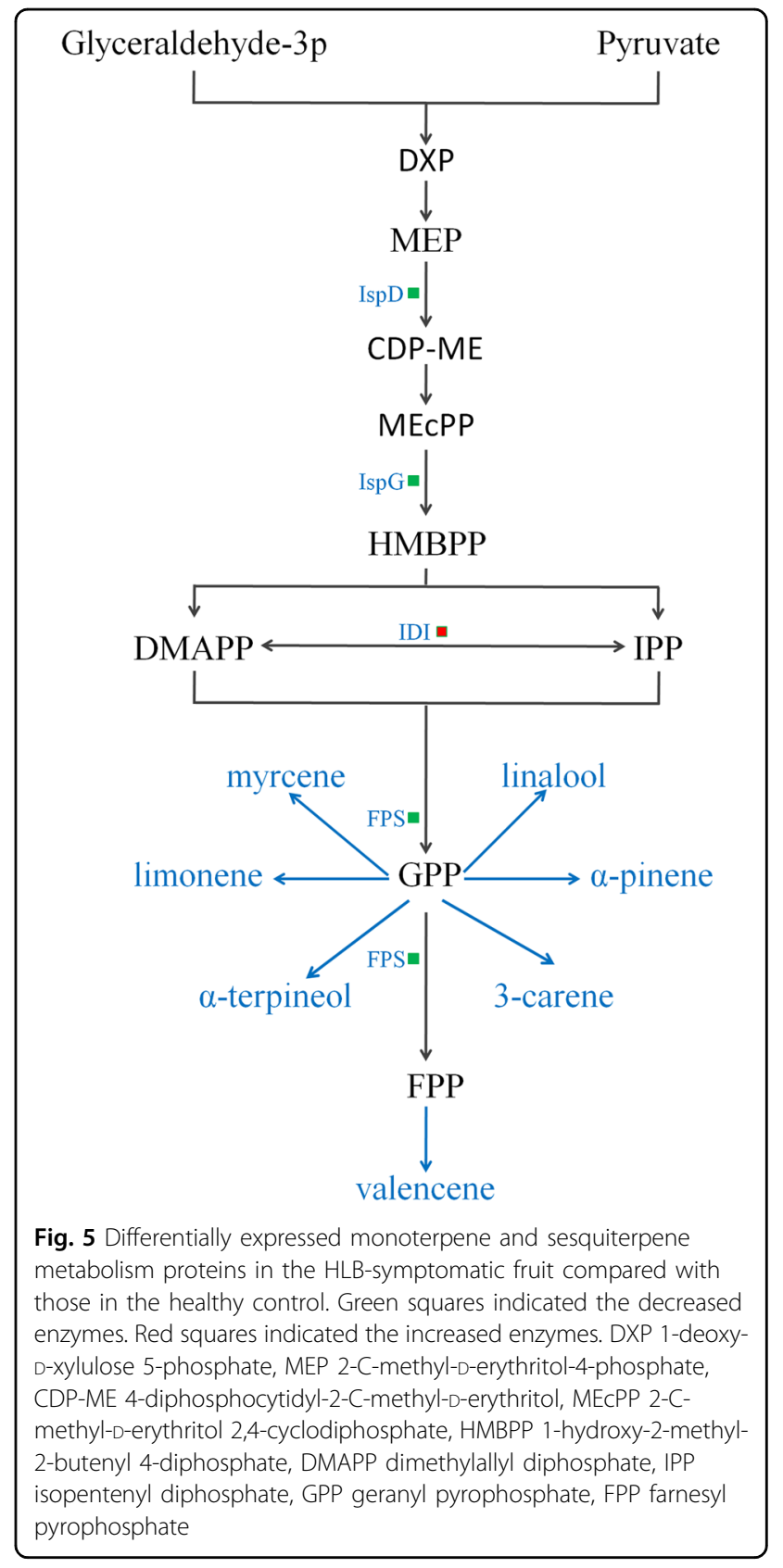

used as a standard for iTRAQ-based quantitative proteomic analysis in tetraploid wheat (Triticum turgidum) ${ }^{18}$ and Flammulina velutipes ${ }^{19}$, two examples where changes in protein expression were mostly $<2$-fold. From a purely technical point of view, it may be thought as a limitation of iTRAQ because of the fold changes in iTRAQ were typically $<2$. However, iTRAQ for quantitative proteomics could provide accurate quantification spanning two orders of magnitude ${ }^{20}$, and the value with false discovery rate $<0.05$ was considered authentic. Although proteins represent the products translated from the transcriptome, 
Table 4 Non-volatile compound content in symptomatic and healthy Valencia orange fruit

\begin{tabular}{|c|c|c|c|c|c|}
\hline TMS derivative $\left(\mu \mathrm{g} \mathrm{g}^{-1}\right)$ & $\mathrm{Rt}(\min )$ & Ion model $(\mathrm{m} / \mathrm{z})$ & Healthy fruits & Symptomatic fruits & $P$-value \\
\hline \multicolumn{6}{|l|}{ Sugars } \\
\hline Arabinose $(\mathrm{TMS})_{4}$ & 29.37 & 307 & $1.99 \pm 0.39$ & $2.36 \pm 1.24$ & $6.44 \mathrm{E}-01$ \\
\hline Fructose $(\mathrm{TMS})_{5}$ & $34.87 / 35.13$ & 307 & $344.81 \pm 14.02$ & $290.44 \pm 35.07$ & $6.73 \mathrm{E}-02$ \\
\hline Glucose $(\mathrm{TMS})_{5}$ & $35.47 / 35.85$ & 319 & $263.22 \pm 10.38$ & $210.59 \pm 20.79$ & $1.72 \mathrm{E}-02$ \\
\hline Mannitol $(\mathrm{TMS})_{6}$ & 36.21 & 319 & $1.33 \pm 0.13$ & $1.84 \pm 0.78$ & $3.31 \mathrm{E}-01$ \\
\hline Myo-inositol $(\mathrm{TMS})_{6}$ & 39.81 & 305 & $102.25 \pm 6.45$ & $96.87 \pm 5.12$ & $3.21 \mathrm{E}-01$ \\
\hline Ribose $(\mathrm{TMS})_{4}$ & 29.79 & 307 & $0.42 \pm 0.03$ & $0.54 \pm 0.11$ & $1.25 \mathrm{E}-01$ \\
\hline Sucrose $(T M S)_{8}$ & 51.29 & 361 & $379.52 \pm 24.37$ & $277.33 \pm 47.93$ & $3.02 \mathrm{E}-02$ \\
\hline L-Xylose $(T M S)_{4}$ & 29.22 & 307 & $3.36 \pm 0.58$ & $2.95 \pm 0.14$ & $3.01 \mathrm{E}-01$ \\
\hline \multicolumn{6}{|l|}{ Organic acids } \\
\hline Citric acid $(\mathrm{TMS})_{4}$ & 33.28 & 273 & $144.80 \pm 8.10$ & $135.38 \pm 11.97$ & 3.60E-01 \\
\hline Fumaric acid $(T M S)_{2}$ & 19.12 & 245 & $0.07 \pm 0.01$ & $0.10 \pm 0.00$ & $3.54 \mathrm{E}-03$ \\
\hline Malic acid $(T M S)_{3}$ & 23.96 & 245 & $66.29 \pm 9.75$ & $60.19 \pm 3.14$ & $3.61 E-01$ \\
\hline Quinic acid $(T M S)_{5}$ & 34.45 & 345 & $13.91 \pm 0.69$ & $16.69 \pm 0.28$ & $2.99 \mathrm{E}-03$ \\
\hline Succinic acid $(T M S)_{2}$ & 18.08 & 247 & $0.32 \pm 0.04$ & $0.49 \pm 0.13$ & 8.30E-02 \\
\hline \multicolumn{6}{|l|}{ Amino acids } \\
\hline D-Alanine $(\mathrm{TMS})_{2}$ & 10.55 & 116 & $0.74 \pm 0.07$ & $0.82 \pm 0.05$ & $1.94 \mathrm{E}-01$ \\
\hline L-Asparagine $(\mathrm{TMS})_{3}$ & 29.13 & 232 & $7.29 \pm 2.94$ & $16.62 \pm 12.73$ & 2.84E-01 \\
\hline L-Aspartic acid $(T M S)_{3}$ & 24.89 & 188 & $9.29 \pm 2.38$ & $8.99 \pm 8.23$ & $9.55 \mathrm{E}-01$ \\
\hline D-Glutamic acid $(T M S)_{3}$ & 27.70 & 246 & $3.45 \pm 0.61$ & $2.98 \pm 1.47$ & $6.34 \mathrm{E}-01$ \\
\hline Glycine $(T M S)_{3}$ & 17.83 & 174 & $0.91 \pm 0.09$ & $1.20 \pm 0.25$ & 1.39E-01 \\
\hline L-Leucine $(T M S)_{2}$ & 16.75 & 158 & $0.04 \pm 0.01$ & $0.07 \pm 0.02$ & 1.07E-01 \\
\hline L-Phenylalanine $(T M S)_{2}$ & 27.64 & 218 & $0.71 \pm 0.14$ & $1.28 \pm 0.59$ & $1.80 \mathrm{E}-01$ \\
\hline L-Proline $(T M S)_{2}$ & 17.43 & 142 & $6.51 \pm 1.21$ & $7.84 \pm 2.16$ & 4.03E-01 \\
\hline L-Serine $(T M S)_{3}$ & 19.91 & 204 & $0.76 \pm 0.03$ & $1.32 \pm 0.36$ & 5.31E-02 \\
\hline L-Threonine $(\mathrm{TMS})_{3}$ & 20.78 & 101 & $0.06 \pm 0.00$ & $0.19 \pm 0.08$ & $5.84 \mathrm{E}-02$ \\
\hline L-Tryptophan $(\mathrm{TMS})_{3}$ & 42.15 & 202 & $0.18 \pm 0.05$ & $0.30 \pm 0.20$ & 3.85E-01 \\
\hline L-Valine $(T M S)_{2}$ & 14.72 & 144 & $0.25 \pm 0.03$ & $0.30 \pm 0.04$ & $1.56 \mathrm{E}-01$ \\
\hline
\end{tabular}

Rt retention time

Table 5 Monoterpenoids and valencene content in symptomatic and healthy Valencia orange fruit

\begin{tabular}{llccr}
\hline Concentration $\left(\boldsymbol{\mu g ~ g}^{-\mathbf{1}}\right)$ & RI & Healthy fruits & Symptomatic fruits & P-value \\
\hline (+)-Valencene & 1023.20 & $1248.06 \pm 486.29$ & $266.31 \pm 67.67$ & $1.06 \mathrm{E}-01$ \\
3-Carene & 1156.40 & $25.17 \pm 0.04$ & $3.22 \pm 0.36$ & $1.35 \mathrm{E}-04$ \\
Limonene & 1202.64 & $42,458.08 \pm 8754.63$ & $7519.50 \pm 2432.43$ & $3.22 \mathrm{E}-02$ \\
Linalool & 1528.46 & $187.30 \pm 30.2$ & $42.02 \pm 22.22$ & $3.17 \mathrm{E}-02$ \\
Myrcene & 1539.75 & $854.79 \pm 94.58$ & $123.69 \pm 44.08$ & $1.00 \mathrm{E}-02$ \\
a-Pinene & 1678.32 & $240.56 \pm 80.62$ & $54.54 \pm 3.86$ & $8.26 \mathrm{E}-02$ \\
a-Terpineol & 1710.00 & $120.47 \pm 21.85$ & $33.53 \pm 2.20$ & $3.04 \mathrm{E}-02$ \\
\hline
\end{tabular}

$R /$ retention index 
protein expression depends only in part on the levels of gene expression.

\section{Host proteins involved in primary metabolism were downregulated in fruit from CaLas-infected trees}

Glycolysis is a central and important metabolic pathway in plants and can provide energy, reducing power, and pyruvate to fuel the TCA cycle, and precursors for amino acid, fatty acid and secondary metabolite biosynthesis ${ }^{21}$. Most of the glycolysis enzymes are upregulated during the development of citrus fruit from the fruit enlargement stage into the fruit maturation and ripening stages ${ }^{22}$. Previous reports have also revealed that the plants in which the glycolysis enzymes were downregulated show significantly retarded growth ${ }^{23-25}$. For CaLas-infected citrus, proteins involved in glycolysis are downregulated in the symptomatic fruits compared with those in healthy fruits ${ }^{1,12,16}$. In our study, 17 proteins involved in the glycolysis pathway were downregulated in orange fruits by CaLas, including two PGKs (gi|568881904 and gi| 568881906) and one PFP (gi|641866581). The downregulation of glycolysis proteins in HLB-affected oranges was likely responsible for production of the smaller and unripened fruits.

\section{Non-volatile and volatile compounds were differentially regulated in fruit from CaLas-infected trees}

When citrus is affected by HLB, Brix and Brix/acid levels are decreased, and the fruit flavour is changed. ${ }^{\circ} \mathrm{Brix}$ is made up of sugars, primarily sucrose, fructose, and glucose. In this study, we found that the contents of sucrose and glucose in the pulp of HLB-symptomatic fruit were decreased significantly when compared with those of the healthy fruit, and the content of fructose and myoinositol also showed a downward trend. The results corroborated the conclusion that the sugar content is reduced in fruit from CaLas-infected trees ${ }^{4,6,7}$. At the same time, we also detected that the content of arabinose, mannitol and ribose in symptomatic fruit increased slightly compared with that in healthy fruit. In symptomatic fruit, malic acid levels were decreased, whereas fumaric acid and succinic levels were increased, which was consistent with the report of Sliz et al. ${ }^{7}$. Citric acid, the dominant organic acid of citrus fruit, was decreased in symptomatic fruit. This finding was similar to the result of Sliz et al. ${ }^{7}$, in which they found that the content of citric acid was decreased in asymptomatic fruit from CaLasinfected trees. Chin et al. ${ }^{6}$ found that quinic acid was increased in symptomatic fruit. We also found that the content of quinic acid, lower than that of citric acid and malic acid, was present at higher levels in the symptomatic fruit when compared with the levels in healthy fruit.

Volatile compounds are produced in oil glands of the peels, leaves, and other citrus plant parts ${ }^{26}$. Combining both mass spectra and retention index value matching, seven terpenes and terpene alcohols were identified. Valencene, a kind of sesquiterpene, is the second most abundant terpene after limonene in orange juice and has been used for many years as a quality and maturity marker ${ }^{27}$. As shown in Table 5, concentration of valencene was lower in symptomatic fruit than in control fruit. This suggests the symptomatic fruit had not matured in a normal way, similar to other reports about the valencene concentrations in HLB-affected orange juice ${ }^{4,8}$. However, the deduced concentration of monoterpenes in the symptomatic fruit pulp was different than that found in other studies. Levels of terpenes, such as linalool, were increased in the orange juice of symptomatic fruit in the published literatures $^{4,8}$. Kiefl et al. ${ }^{8}$ reported that the juice oil levels were similar in HLB-affected orange juice (174 $\left.\mathrm{mg} \mathrm{kg}^{-1}\right)$ and healthy control orange juice $\left(185 \mathrm{mg} \mathrm{kg}^{-1}\right)$. They also found $\mathrm{CaLas}$ infection induces significantly higher levels of peel oil aroma volatiles. Some kinds of volatile biomolecules, such as (Z)-4-ecenal and (E,E)-2,4decadienal, could pass into the juice from the peel, and increase concentrations in the orange juice by 38 and 19\%, respectively ${ }^{28,29}$. This indicated that in the process of squeezing juice, the increased volatiles in HLB-affected peel oil were transferred into the juice and might change the concentration of volatiles in juice. In our study, we removed the peel and measured the monoterpene concentration in the fruit pulp through GC-MS and found that they were reduced in the juice sacs of symptomatic fruits. The output of healthy orange juice with commercially acceptable quality has been decreasing year by year since HLB has attacked the Florida citrus industry. It may be possible to utilise some of the volatile aromatic metabolites found in healthy fruit peel to improve the flavour of processed orange juice.

\section{Correlation between the downregulation of proteins and decreased terpenoid levels}

Studies on the pathogenic mechanism of HLB have shown that $\mathrm{Ca}$ Las infection induces the production of callose deposition in plants, causing the obstruction of phloem and impeding the transport of organic compounds from source to sink, which results in starch deposition and mottled leaves ${ }^{30,31}$. In citrus, sucrose is translocated to the fruits from the leaves throughout fruit development and constitutes approximately $50 \%$ of the total soluble sugar ${ }^{32}$. The significantly reduced sucrose in the symptomatic fruits might be the result of a block from the leaves (source) into the fruits (sink) induced by the CaLas infection ${ }^{15,30}$. Imported sucrose in sink organs can be degraded to fructose and glucose by invertase or to fructose and UDP-glucose by SUS. We did not find change in the levels of invertase. However, an SUS, SUS3 (gi|641868466), was downregulated in the symptomatic 
fruits compared with healthy fruits. SUS3 expression was confirmed in the sink tissues of Arabidopsis ${ }^{33}$. Interestingly, fructose content in the same fruits was reduced because of the decreased levels of sucrose and downregulated SUS (Table 4).

Glyceraldehydes-3-P and pyruvate were the intermediate products in the glycolysis process, providing the carbon skeleton for terpenoid compound biosynthesis in the fruit. In this process, six proteins catalyzing the transformation from glucose and fructose to glyceraldehyde-3-P were present at lower levels in the HLB-symptomatic fruits than in the healthy fruits (Fig. 3). Two PGIs (gi|641862435 and gi|641865848) transformed glucose-6P to fluctose-6P, two FRKs (gi|568849997 and gi|641841250) phosphorylated fructose, PFP (gi| 641866581) and FBA (gi|568828917) catalysed fructose$6 \mathrm{P}$ to glyceraldehyde-3P. Pyruvate was obtained from dephosphorylated phosphoenolpyruvate through PK (gil 641842093) and from a non-TCA-cyclic flux compound, malate, via ME (gi|641867382) catalysis. The pyruvate precursor, phosphoenolpyruvate, was obtained from glycerate-2P catalysed by enolases (gi|568856679, gi| 568864726, and gi|568870518) and from oxaloacetate catalysed by PCK (gi|568836722). The levels of these proteins associated with pyruvate metabolism were decreased in the HLB-symptomatic fruit compared with those in the healthy fruit (Fig. 3). Symptomatic fruit was similar to immature fruit, and the PCK and ME increase in healthy fruit agreed with the determination that these proteins accumulate during fruit development and ripening ${ }^{22}$.

Terpenoids have many volatile representatives and compose the largest group of plant secondary metabolic compounds. IPP and its allylic isomer DMAPP are the five universal carbon precursors for all terpenoid products. In the plastids, IPP is synthesised from pyruvate and glyceraldehyde-3-phosphate via the MEP pathway, in which there are seven subsequent enzymatic steps. The third enzyme, IspD (gi|568833221), and sixth enzyme, IspG (gi|568869804), involved in the conversion of MEP into CDP-ME, and MEcPP into HMBPP, respectively ${ }^{34}$, were downregulated in the symptomatic fruit (Fig. 5). IspG overexpression could lead to accumulation of intermediate $\mathrm{HMBPP}^{35}$. The reduced levels of IspD and IspG in HLB fruit might affect the production of HMBPP.

IDI1 (type I IPP isomerase) is mainly found in the plastid and converts IPP to DMAPP ${ }^{36}$. Pankratov et al. ${ }^{37}$ determined that mutations of IDI1 in tomato could reduce overall carotenoid accumulation in fruits. Carotene cleavage dioxygenases $(\mathrm{CCD})$ catalyse the cleavage of carotenoids to apocarotenoids. Overexpression of AtCCD1 could induce $\beta$-ionone emission ${ }^{38}$, whereas CCD1 loss-of-function mutants in Arabidopsis showed an increase in their seed carotenoid levels ${ }^{39}$. IDI1 (gi)
641835071) was upregulated, and CCD1 (gi|568858669) (Table 3) was downregulated in the symptomatic orange fruit, which might lead to carotenoid accumulation and terpenoids reduction in the fruit pulp. CaLas appears to accelerate the biosynthesis of carotenoid pigments in HLB-affected Valencia sweet orange leaves ${ }^{40}$.

FPS catalyses consecutive $E$-condensations of two IPPs with one DMAPP to form the intermediate GPP, then form the end product FPP. GPP is catalysed to form an array of monoterpenes ${ }^{41}$, and FPP is catalysed to form sesquiterpenes and di-, tri-, and tetra-terpenes in high to low organisms. FPS is considered as a rate-limiting enzyme that determines the flow rate of GPP and $\mathrm{FPP}^{42}$. Overexpression of the FPS2 protein could increase terpenoid accumulation in plants ${ }^{43}$. FPS2 (gi|641840281) was downregulated in the HLB-symptomatic fruit, and the concentration of monoterpenes and valencene were decreased in the pulp of these fruits compared with that of healthy controls.

\section{Conclusions}

The results from studies performed on citrus fruit pulp from $\mathrm{Ca}$ Las-infected trees revealed significant suppression and metabolic dysfunction in sugar and organic acid metabolism and homeostasis, and terpenoid metabolism. These cause major disruptions in the production of proteins, sugars, organic acids, and volatiles, resulting in lower levels of sugars and terpenoids in symptomatic fruit pulp, and thereby causes many of the negative attributes found in juice produced from such fruit.

\section{Materials and methods \\ Materials}

The harvest season for Valencia orange covers 4 months, typically from late-February or early-March, to late-May or early-June. Fruit harvested in the middle to late months have the highest concentrations of many important aroma compounds, compared with those harvested in the very early or late months of the Valencia maturity season ${ }^{44}$. Fruits of 8-year-old Valencia orange (Citrus sinensis) scions on Swingle citrumelo rootstocks were harvested from a commercial citrus grove in St. Cloud, Florida, USA, on 1 April 2015. Because of the widespread incursion of HLB in Florida and in this specific grove as well, we found only two PCR-negative and healthy trees. Consequently, biological sample replication was restricted to just two healthy trees to use as controls, for comparison with three replicates of symptomatic trees. Symptomatic and healthy fruits were harvested from PCR-positive and PCR-negative trees, respectively. Five fruits randomly selected from one tree were peeled, and pulp was mixed as one biological replicate. The peeled pulp of fruits was stored at $-80^{\circ} \mathrm{C}$. 
Analytical grade standards of non-volatile polar compounds; volatiles; internal standards, including 4-heptadecanone and ribitol, n-alkanes $(\mathrm{C} 8-\mathrm{C} 20)$ and chemical reagents; and dichloromethane, pyridine, methoxyamine hydrochloride (MEOX) and $\mathrm{N}$-methyl- $\mathrm{N}$ (trimethylsilyl)-trifluoroacetamide (MSTFA) were purchased from Sigma (Sigma-Aldrich Co., St. Louis, MO, USA).

\section{Protein extraction}

Total protein was extracted using a phenol method ${ }^{45}$. A total of $5 \mathrm{~g}$ of frozen juice sac samples were ground in liquid nitrogen, and $12 \mathrm{~mL}$ of protein extraction buffer (0.5 M Tris (pH 7.5), $0.1 \mathrm{M} \mathrm{KCl,} 0.7 \mathrm{M}$ sucrose, $0.05 \mathrm{M}$ EDTA, $0.05 \mathrm{M}$ dithiothreitol, $1 \mathrm{mM}$ phenylmethanesulfonyl fluoride, $1 \%$ 2-mercaptoethanol, and $0.1 \%$ protease inhibitor mix and $18 \mathrm{~mL}$ Trissaturated phenol) was added. After three more rounds of phenol extraction, the top phenol phase was moved into a new tube and $30 \mathrm{~mL}$ of ammonium acetate methanol solution was poured into the tube to precipitate the pellet. The pellet was formed by centrifugation and washed twice, using $10 \mathrm{~mL}$ ice cold $100 \%$ methanol for one wash and ice cold $100 \%$ acetone for the second wash.

\section{Proteomic ITRAQ and data analysis}

For the iTRAQ experiments, five specimens were labelled and analysed (three biological replicates of the symptomatic sample and two biological replicates of the healthy sample). The procedutres were performed according to the manufacturer's protocols at the Interdisciplinary Center for Biotechnology Research (ICBR) of the University of Florida (Gainesville, FL, USA). For protein identification, the ProteinPilot v5.0 software integrated with Paragon ${ }^{\mathrm{mm}}$ algorithm (https://sciex.com/ products/software/proteinpilot-software) was employed against the GenBank subset of $C$. sinensis plants in the FASTA formation (downloaded in December 2012), using the original MS/MS data for database searching. The statistical analysis for relative quantification of proteins was performed using the Pro Group ${ }^{\mathrm{Tm}}$ Algorithm embedded in the ProteinPilot Software. A differentially expressed protein had to be quantified with a fold change $>1.5$ or $<0.67$ and a $P$-value $<0.05$. Arabidopsis orthologues were determined for each differentially expressed protein through local blastx ( $e$-value $<$ $10^{-3}$ ) to the predicted Arabidopsis proteins in the TAIR database (TAIR10_pep_20101028). The biological interpretations of the relatively quantified proteins were obtained by attributing them to their own metabolic pathways with Kyoto Encyclopaedia of Genes and Genomes (KEGG) annotation and MAPMAN 3.6.0RC1 software ${ }^{46}$.

\section{Preparation of the aroma isolates}

A total of $50 \mathrm{~g}$ of pulp frozen in liquid nitrogen was ground and transferred into a plastic bottle; $100 \mathrm{~mL}$ of distilled dichloromethane was added, and the pulp powder was extracted twice. The supernatant was filtered using filter paper, and $250 \mu \mathrm{L}$ of $2 \mathrm{mg} \mathrm{mL}^{-1}$ 4-heptadecanone was added. The volatile compounds were isolated using the solvent-assisted flavour evaporation extraction method and concentrated to $150 \mu \mathrm{L}$ according to Huang's description ${ }^{47}$.

\section{Preparation and TMS derivatization of non-volatile metabolites}

Non-volatile organic compounds were extracted and derivatized according to Lisec's protocol ${ }^{48}$, although several modifications were made. A total of $20 \mathrm{~g}$ pulp frozen in liquid nitrogen was ground to powder and transferred to a plastic bottle, and $60 \mathrm{~mL}$ of $100 \%$ distilled methanol (precooled at $-20^{\circ} \mathrm{C}$ ) was added into the bottle and vortexed for $10 \mathrm{~s}$; then, $1 \mathrm{~mL}$ of $10 \mathrm{mg} \mathrm{mL}^{-1}$ ribitol was added as an internal quantitative standard. The bottle was shaken for $30 \mathrm{~min}$ at $70^{\circ} \mathrm{C}$ and centrifuged for $10 \mathrm{~min}$ at $11,000 \mathrm{~g}$ at room temperature. The upper phase was moved to a new glass bottle and evaporated to remove water using a rotary evaporator and exhaustively dried under vacuum conditions using a freeze dryer (Labconco FreeZone 2.5, Kansas City, MO, USA). A total of $10 \mathrm{mg}$ freeze-dried powder was added, along with $80 \mu \mathrm{L}$ of methoxyamination reagent, to the aliquots; $40 \mu \mathrm{L}$ of the solution was transferred into a new tube with another 40 $\mu \mathrm{L}$ of methoxyamination reagent and shaken for $2 \mathrm{~h}$ at 37 ${ }^{\circ} \mathrm{C}$. A total of $70 \mu \mathrm{L}$ MSTFA solution was added to the sample aliquots and shaken for $30 \mathrm{~min}$ at $37^{\circ} \mathrm{C}$ again. Then, the liquid was filtered and transferred into glass vials and run on the GC for no $>24 \mathrm{~h}$.

\section{GC-MS analysis of volatile compounds}

GC was performed on a PerkinElmer Clarus 680 (PerkinElmer, Inc., MA, USA) equipped with an autosampler. A trace-free fatty acid phase column $(30 \mathrm{~m} \times 0.25 \mathrm{~mm}$, $0.25 \mu \mathrm{m}$ film thickness) was used. MS analysis was performed on a PerkinElmer Clarus SW8T (PerkinElmer).

Helium carrier gas was used at a flow rate (constant mode) of $5.0 \mathrm{~mL} \mathrm{~min}^{-1}$. The oven temperature ramp for the GC analysis of volatile compounds was as follows: the initial oven temperature was $40^{\circ} \mathrm{C}$, held for $1 \mathrm{~min}$; then heating at $30^{\circ} \mathrm{C} \mathrm{min}^{-1}$ up to $100^{\circ} \mathrm{C}$, held for $2 \mathrm{~min}$; at the end heating at $5^{\circ} \mathrm{C} \mathrm{min}{ }^{-1}$ up to $230{ }^{\circ} \mathrm{C}$, held for $10 \mathrm{~min}$. It took $41 \mathrm{~min}$ for the entire run.

\section{GC-MS analysis for TMS derivatives}

GC-MS analysis was performed on an Agilent GC-MS 5975C-7890A (Agilent Technologies, CA, USA) with an autosampler (7693). A Rxi-5 MS column (Crossbond 
diphenyl dimethyl polysiloxane; $30 \mathrm{~m} \times 0.25 \mathrm{~mm}, 0.25 \mu \mathrm{m}$ film thickness) from Restek (Cat\#:13423, Restek Corporation, Bellefonte, PA, USA) was used.

The carrier gas, helium, ran at a flow rate (constant mode) of $1.1 \mathrm{~mL} \mathrm{~min}^{-1}$. In all, $1 \mu \mathrm{L}$ of TMS derivatives was injected into an injector with temperature $230{ }^{\circ} \mathrm{C}$ and split ratio 10:1. The oven temperature ramp for the GC analysis of volatile compounds was as follows: initial temperature $70^{\circ} \mathrm{C}$, held for $5 \mathrm{~min}$; then heating at $4{ }^{\circ} \mathrm{C}$ $\min ^{-1}$ to $270^{\circ} \mathrm{C}$, at the end $20^{\circ} \mathrm{C} \mathrm{min}^{-1}$ to $320^{\circ} \mathrm{C}$ for 4.5 min. It took $62 \mathrm{~min}$ for the entire run. The fixed MS settings were scan mode for the analysis of the TMS derivatives, full with a mass range $\mathrm{m} / \mathrm{z} 60-650$.

MS peak identification and internal standard quantification

GC-MS chromatograms were analysed using TurboMass software version 5.4.2 (PerkinElmer, Waltham, MA, USA). Peaks were first identified by comparing their mass spectra with the NIST library (National Institute of Standards and Technology, Gaithersberg, MA, USA) and 9th edition Wiley (John Wiley and Sons, Inc., Hoboken, NJ, USA). Identified results from libraries were further confirmed, after comparing the retention time and mass spectra of derivatized standards with those of the detected chemical compounds. 4-Heptadecanone was used as an internal standard for the analysis of volatile compounds, and ribitol acted as an internal standard for non-volatile compounds. Semiquantitative measurements were implemented for the metabolite concentrations.

\section{Statistical analysis of metabolic compounds}

Pooled samples from five randomly selected fruits were used for each individual biological replicated tree. The differences of metabolic compounds between healthy and symptomatic fruits were examined by an analysis of variance using the JMP 12.0 statistical package (SAS Institute, Cary, NC, USA). A two-sample $t$-test was performed to assess the significance of differences for specific metabolic compounds between healthy and symptomatic fruits.

\section{Acknowledgements}

We thank Tony Trama (Florida Department of Citrus, FL) for providing the GCMS instrument for non-volatile compound analysis. This work was supported by the Florida Citrus Research and Development Foundation (CRDF), the Earmarked Fund for China Agriculture Research System (CARS-27), and China Scholarship Council.

\section{Authors' contributions}

L.Y. and Q.Y. designed the experiments. L.Y. carried out the experiment, analysed all data, and wrote the draft manuscript. M.H. participated in ITRAQ analysis and W.H. ran GC-MS for non-volatile compounds. J.G. and S.C. took part in data analysis. Q.Y. and Y.W. assisted in the preparation of the manuscript. F.G.G. supervised the overall study and revised the whole manuscript. All authors have read and approved the final version of the manuscript.
Conflict of interest

The authors declare that they have no conflict of interest.

\section{Publisher's note}

Springer Nature remains neutral with regard to jurisdictional claims in published maps and institutional affiliations.

Received: 23 August 2018 Revised: 11 November 2018 Accepted: 15 November 2018

Published online: 14 February 2019

\section{References}

1. Liao, H. L. \& Burns, J. K. Gene expression in Citrus sinensis fruit tissues harvested from Huanglongbing-infected trees: comparison with girdled fruit. J. Exp. Bot. 63, 3307-3319 (2012)

2. Tatineni, S. et al. In planta distribution of 'Candidatus Liberibacter asiaticus' as revealed by polymerase chain reaction (PCR) and real-time PCR. Phytopathology 98, 592-599 (2008).

3. Bové, J. M. \& Ayres, A. J. Etiology of three recent diseases of citrus in São Paulo State: Sudden death, variegated chlorosis and Huanglongbing. IUBMB Life 59, 346-354 (2007).

4. Baldwin, E. et al. Effect of Liberibacter infection (Huanglongbing disease) of citrus on orange fruit physiology and fruit/fruit juice quality: chemical and physical analyses. J. Agric. Food Chem. 58, 1247-1262 (2010).

5. Dagulo, L. et al. Chemical characterization of orange juice from trees infected with citrus greening (Huanglongbing). J. Food Sci. 75, C199-C207 (2010).

6. Chin, E. L., Mishchuk, D. O., Breksa, A. P. \& Slupsky, C. M. Metabolite signature of Candidatus Liberibacter asiaticus infection in two citrus varieties. J. Agric. Food Chem. 62, 6585-6591 (2014).

7. Slisz, A. M., Breksa, A. P. 3rd, Mishchuk, D. O., McCollum, G. \& Slupsky, C. M. Metabolomic analysis of citrus infection by 'Candidatus Liberibacter' reveals insight into pathogenicity. J. Proteome Res. 11, 4223-4230 (2012).

8. Kiefl, J. et al. Investigation on key molecules of Huanglongbing (HLB)-induced orange juice off-flavor. J. Agric. Food Chem. 66, 2370-2377 (2018).

9. Paula, B. M. D. et al. Active taste compounds in juice from oranges symptomatic for Huanglongbing (HLB) citrus greening disease. LWT-Food Sci. Technol. 91, 518-525 (2018).

10. Wang, Y. et al. Transcriptome profiling of Huanglongbing $(H L B)$ tolerant and susceptible citrus plants reveals the role of basal resistance in HLB tolerance. Front. Plant Sci. 7, 933 (2016).

11. Martinelli, F., Reagan, R. L., Dolan, D., Fileccia, V. \& Dandekar, A. M. Proteomic analysis highlights the role of detoxification pathways in increased tolerance to Huanglongbing disease. BMC Plant Biol. 16, 167 (2016).

12. Zhong, Y. et al. Digital gene expression analysis of Ponkan mandarin (Citrus reticulata Blanco) in response to Asia citrus psyllid-vectored Huanglongbing infection. Int. J. Mol. Sci. 17, 1063 (2016).

13. Albrecht, U., Fiehn, O. \& Bowman, K. D. Metabolic variations in different citrus rootstock cultivars associated with different responses to Huanglongbing. Plant Physiol. Biochem. 107, 33-44 (2016).

14. $\mathrm{Yu}, \mathrm{Q}$. et al. Reprogramming of a defense signaling pathway in rough lemon and sweet orange is a critical element of the early response to 'Candidatus Liberibacter asiaticus'. Hortic. Res. 4, 17063 (2017).

15. Rosales, R. \& Burns, J. K. Phytohormone changes and carbohydrate status in sweet orange fruit from Huanglongbing-infected trees. Plant Growth Regul. 30 312-321 (2011).

16. Martinelli, F. et al. Transcriptome profiling of citrus fruit response to Huanglongbing disease. PLoS One 7, e38039 (2012).

17. Li, W., Hartung, J. S. \& Levy, L. Quantitative real-time PCR for detection and identification of Candidatus Liberibacter species associated with citrus Huanglongbing. J. Microbiol. Methods 66, 104-115 (2006).

18. Chen, S., Chen, J, Hou, F, Feng, Y. \& Zhang, R. iTRAQ-based quantitative proteomic analysis reveals the lateral meristem developmental mechanism for branched spike development in tetraploid wheat (Triticum turgidum L.). BMC Genom. 19, 228 (2018)

19. Liu, J. Y., Men, J. L., Chang, M. C., Feng, C. P. \& Yuan, L. G. iTRAQ-based quantitative proteome revealed metabolic changes of Flammulina velutipes mycelia in response to cold stress. J. Proteom. 156, 75-84 (2017). 
20. OW, S. Y. et al. iTRAQ underestimation in simple and complex mixtures: "The good, the bad and the ugly". J. Proteome Res. 8, 5347-5355 (2009).

21. Plaxton, W. C. The organization and regulation of plant glycolysis. Annu. Rev. Plant Physiol. Plant Mol. Biol. 47, 185-214 (1996).

22. Katz, E. et al. Label-free shotgun proteomics and metabolite analysis reveal a significant metabolic shift during citrus fruit development. J. Exp. Bot. 62 5367-5384 (2011).

23. Eremina, M., Rozhon, W., Yang, S. \& Poppenberger, B. ENO2 activity is required for the development and reproductive success of plants and is feedbackrepressed by AtMBP-1. Plant J. 81, 895-906 (2015)

24. Rosa-Téllez, S. et al. Phosphoglycerate kinases are co-regulated to adjust metabolism and to optimize growth. Plant Physiol. 176, 1182-1198 (2018).

25. Chen, M. \& Thelen, J. J. The plastid isoform of triose phosphate isomerase is required for the postgerminative transition from heterotrophic to autotrophic growth in Arabidopsis. Plant Cell 22, 77-90 (2010).

26. Maffei, M. E., Jürgens, A. \& Viljoen, A. M. Sites of synthesis, biochemistry and functional role of plant volatiles. S. Afr. J. Bot. 76, 612-631 (2010).

27. Elston, A., Lin, J. \& Rouseff, R. Determination of the role of valencene in orange oil as a direct contributor to aroma quality. Flavour Fragr. J. 20, 381-386 (2010)

28. Buettner, A. \& Schieberle, P. Evaluation of aroma differences between handsqueezed juices from Valencia late and Navel oranges by quantitation of key odorants and flavor reconstitution experiments. J. Agric. Food Chem. 49, 2387-2394 (2001).

29. Seideneck, R. \& Schieberle, P. Comparison of the key aroma compounds in hand-squeezed and unpasteurised, commercial NFC juices prepared from Brazilian Pera Rio oranges. Eur. Food Res. Technol. 232, 995-1005 (2011).

30. Kim, J. S., Sagaram, U. S., Burns, J. K., Li, J. L. \& Wang, N. Response of sweet orange (Citrus sinensis) to 'Candidatus Liberibacter asiaticus' infection: microscopy and microarray analyses. Phytopathology 99, 50-57 (2009).

31. Etxeberria, E., Gonzalez, P., Achor, D. \& Albrigo, G. Anatomical distribution of abnormally high levels of starch in HLB-affected Valencia orange trees. Physiol. Mol. Plant Path. 74, 76-83 (2009).

32. Sauer, N. Molecular physiology of higher plant sucrose transporters. FEBS Lett. 581, 2309-2317 (2007).

33. Ruan, M. B., Liao, W. B., Zhang, X. C., Yu, X. L. \& Peng, M. Analysis of the cotton sucrose synthase 3 (Sus3) promoter and first intron in transgenic Arabidopsis. Plant Sci. 176, 342-351 (2009).

34. Hsieh, M. H., Chang, C. Y., Hsu, S. J. \& Chen, J. J. Chloroplast localization of methylerythritol 4-phosphate pathway enzymes and regulation of mitochondrial genes in ispD and ispE albino mutants in Arabidopsis. Plant Mol. Biol. 66, 663-673 (2008).
35. Li, Q. et al. Balanced activation of IspG and IspH to eliminate MEP intermediate accumulation and improve isoprenoids production in Escherichia coli. Metab. Eng. 44, 13-21 (2017).

36. Phillips, M. A., D'Auria, J. C., Gershenzon, J. \& Pichersky, E. The Arabidopsis thaliana type I isopentenyl diphosphate isomerases are targeted to multiple subcellular compartments and have overlapping functions in isoprenoid biosynthesis. Plant Cell 20, 677-696 (2008).

37. Pankratov, I. et al. Fruit carotenoid-deficient mutants in tomato reveal a function of the plastidial isopentenyl diphosphate isomerase (IDI1) in carotenoid biosynthesis. Plant J. 88, 82-94 (2016).

38. Cáceres, L. A. et al. Repellent and attractive effects of $\alpha-$, $\beta$-, and dihydro- $\beta$ ionone to generalist and specialist herbivores. J. Chem. Ecol. 42, 107-117 (2016).

39. Auldridge, $M$. et al. Characterization of three members of the Arabidopsis carotenoid cleavage dioxygenase family demonstrates the divergent roles of this multifunctional enzyme family. Plant J. 45, 982-993 (2006).

40. Killiny, N. \& Nehela, Y. One target, two mechanisms: the impact of Candidatus Liberibacter asiaticus and its vector, Diaphorina citri on citrus leaf pigments. Mol. Plant Microbe Interact. 30, 543-556 (2017).

41. Zebec, Z. et al. Towards synthesis of monoterpenes and derivatives using synthetic biology. Curr. Opin. Chem. Biol. 34, 37-43 (2016).

42. Szkopińska, A. \& Płochocka, D. Farnesyl diphosphate synthase; regulation of product specificity. Acta Biochim. Pol. 52, 45-55 (2005).

43. Bhatia, V., Maisnam, J., Jain, A., Sharma, K. \& Bhattacharya, R. Aphid-repellent pheromone $\mathrm{E}-\beta$-farnesene is generated in transgenic Arabidopsis thaliana over-expressing farnesyl diphosphate synthase2. Ann. Bot. 115, 581-591 (2015)

44. Bai, J. et al. Changes in volatile and non-volatile flavor chemicals of "Valencia" orange juice over the harvest seasons. Foods 5, 4 (2016).

45. Rode, C. et al. Enolases: storage compounds in seeds? Evidence from a proteomic comparison of zygotic and somatic embryos of Cyclamen persicum Mill. Plant Mol. Biol. 75, 305-319 (2011).

46. Thimm, O. et al. MapMan: a user-driven tool to display genomics data sets onto diagrams of metabolic pathways and other biological processes. Plant $\mathrm{J}$. 37, 914-939 (2004).

47. Huang, M. et al. Characterization of the major aroma-active compounds in peel oil of an HLB-tolerant Mandarin hybrid using aroma extraction dilution analysis and gas chromatography-mass spectrometry/olfactometry. Chem. Percept. 10, 161-169 (2017).

48. Lisec, J., Schauer, N., Kopka, J., Willmitzer, L. \& Fernie, A. R. Gas chromatography mass spectrometry-based metabolite profiling in plants. Nat. Protoc. 1, 387-396 (2006) 